Ionic Core Effects on the Mie Resonance in Lithium Clusters

K. Yabana ${ }^{(a)}$ and G. F. Bertsch(b)

(a) Department of Physics, Niigata University, Niigata 950-21, Japan and

(b) Department of Physics and Institute for Nuclear Theory, University of Washington, Seattle, Washington, 98195

PREPARED FOR THE U.S. DEPARTMENT OF ENERGY UNDER GRANT DE-FG06-90ER40561

This report was prepared as an account of work sponsored by the United States Government. Neither the United States nor any agency thereof, nor any of their employees, makes any warranty, express or implied, or assumes any legal liability or responsibility for the accuracy, completeness, or usefulness of any information, apparatus, product, or process disclosed, or represents that its use would not infringe privately owned rights. Reference herein to any specific commercial product, process, or service by trade name, mark, manufacturer, or otherwise, does not necessarily constitute or imply its endorsement, recommendation, or favoring by the United States Government or any agency thereof. The views and opinions of authors expressed herein do not necessarily state or reflect those of the United States Government or any agency thereof.

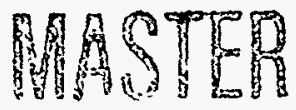

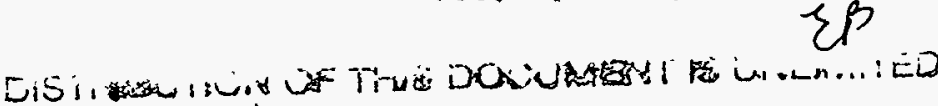




\section{DISCLAIMER}

Portions of this document may be illegible in electronic image products. Images are produced from the best available original document. 


\title{
Ionic core effects on the Mie resonance in lithium clusters
}

\author{
K. Yabana $a^{(a)}$ and G.F. Bertsch ${ }^{(a)}$ \\ ${ }^{(a)}$ Department of Physics, Niigata University, \\ Niigata 950-21, Japan \\ ${ }^{(b)}$ Department of Physics and National Institute for Nuclear Theory, \\ University of Washington \\ Seattle, WA 98195
}

\begin{abstract}
We investigate the effects of the atomic cores on the Mie resonance in lithium metal clusters, perturbing a jellium Hamiltonian with zero-range pseudopotentials. The resonance is red-shifted with respect to the classical formula by core effects, most important of which is the increased effective mass due to the core potentials. Much of the large shift seen in lithium clusters is thereby explained if the strength of the pseudopotentials is taken from band structure calculations. However, such pseudopotentials cause the resonance to be greatly broadened, contrary to observation.
\end{abstract}

\section{INTRODUCTION}

A prominent feature of the response of alkali metal clusters [1] is a strong concentration of strength at a frequency near that of the classical Mie resonance,

$$
\omega_{M}=\sqrt{\frac{e^{2} N}{m R^{3}}}
$$

Here $N$ is the number of valence electrons, $R$ is the radius of the cluster, and $e$ and $m$ are the electronic charge and mass, respectively. The observed resonance frequency is always lower 
in alkali metals than the classical value, however. This red shift is particularly prominent in lithium clusters [2], where it amounts to $\sim 27 \%$.

The origin of the red shift has been somewhat of a puzzle to cluster theorists. The shift is properly reproduced in quantum chemistry calculations $[3,4]$, but these studies give no clue as to the physical origin of the shift, and the technique is limited to small clusters. For larger systems, the response must be calculated in a more tractable theory, such as linear response with the local density approximation, also known as RPA. Using the RPA in a jellium approximation, one finds a strong concentration of strength slightly red-shifted from the Mie formula. The reason for the shift is the spill-out effect, which is that the effective electron radius is larger than the jellium core radius $R$. In effect, the $R$ in eq. (1) should be increased slightly because of the single-electron quantum mechanics.

There are other physical perturbations on the jellium picture which affect the Mie resonance. The core electrons, which in the jellium model are frozen into the ionic charge, can polarize and give the environment of the valence electrons a dielectric constant larger than one. This effect is estimated in Appendix A; it is small except for the heaviest alkali metal, Cs. In particular, the core polarization is negligible for the metal with the largest Mie red shift, namely Li.

In this work we will consider the effect of the ionic cores on the resonant frequency in lithium clusters. In the metal there is a large red shift in the measured plasmon resonance [5] which may be interpreted as a band structure enhancement of the effective mass [6]. Effects of ionic core potentials have previously been considered on the structure of metal clusters [7] and on their Mie resonance [8,9]. However, ref. [8] and [9] approximate the core potential by a spherical average. This may leave out important lattice effects, as we shall argue in the next section. Our approach will be to take a simple model for the pseudopotential, but to treat the lattice structure explicitly. 


\section{CORE POTENTIAL EFFECTS}

The core potentials act to increase the effective mass of the electrons. Naively looking at eq. (1), an effective mass enhancement should produce a red-shift for the Mie resonance. To capture the physics of the effective mass enhancement, we should should first understand how it comes about [10]. There are two reasons why the core potential increases the effective mass in the metallic lattice. Fundamentally, the requirement that the wave functions of the conduction electrons be orthogonal to those of core electron decreases the phase available to the conduction electron. The restriction is stronger for low-momentum states in the conduction band than for high-momentum states, raising the energy of low-momentum states. This is included in the usual treatment of band structure with pseudopotentials; this effect appears because the pseudopotential is repulsive for $s$-waves but attractive for $p$-waves. The states in the band have mainly $s$-wave character at low momentum, and acquire more $p$-wave character at higher momentum.

There is a second reason for the effective mass enhancement which is more directly associated with the lattice regularity. Consider the single-particle energy-momentum dispersion curve, sketched in Fig. 1 for a large system. In the jellium model, the energies are pure single-particle kinetic energies, given by $\hbar^{2} k^{2} / 2 m$. This is indicated by the dashed line. The ionic cores give rise to a band gap, perturbing the energy levels as depicted. For an alkali metal, the Fermi momentum is somewhat below the the band edge. The important point is that the slope of the dispersion curve at the Fermi momentum is decreased by the perturbation. The band energy will therefore have an effective mass $m^{*}>m$. Putting this into eq. (1), the resonance would be red-shifted.

The first effect, a smooth momentum dependence of the ionic core potential, can be estimated rather simply considering the ionic cores as a perturbation on the jellium singleelectron Hamiltonian. This is done in Appendix B. For lithium, the effective mass obtained this way is about $1.3 \mathrm{~m}$. Effective masses in this range may also be inferred from band structure calculations of the single particle energies. For example, Table IV of ref. [10] gives 
a fit to the smooth part of the energy dispersion, which implies an effective mass of $1.3 \mathrm{~m}$. Similar dispersion curves may be found in refs. $[6,15]$.

The lattice regularity also induces an enhanced effective mass. When the Fermi surface is near the edge of the Brillouin zone, the coherent mixing with nearby states in the next zone increases the level density as well as producing the band gap. The band calculation of Ref. [15] gives 6.73 states/Ry/atom at the Fermi surface, implying an effective mass $m^{*}=1.56 m$.

Ref. [6] quotes an optical effective mass of 1.48. The difference between these numbers and the smaller effective mass in the previous paragraph indicates the importance of the specific lattice structure.

It is not possible to include a realistic atomic core potential in the calculation of any but the smallest finite clusters, due to computational limitations. We shall therefore use a very simplified pseudopotential to study the effects of the ion cores in moderate size clusters. Since we consider the perturbation from the jellium model to be small, the detailed form of the pseudopotential should be irrelevant if its momentum dependence is treated properly. We are thus led to consider momentum-dependent contact interactions. With $s$ - and $p$-wave perturbations treated separately, the explicit form of the pseudopotential is

$$
v=b_{0} \delta(r)+b_{1} \nabla \cdot \delta(r) \nabla+\frac{b_{2}}{2}\left(\nabla^{2} \delta(r)+\delta(r) \nabla^{2}\right)
$$

This potential has plane-wave matrix elements given by

$$
\left\langle\vec{k}|v| \overrightarrow{k^{\prime}}\right\rangle=b_{0}+b_{1} k \cdot k^{\prime}+\frac{b_{2}}{2}\left(k^{2}+k^{2}\right)
$$

Using the $T$-matrix interaction of Appendix B, we obtain values for the $b$ parameters given in Table I. However, the $T$-matrix calculation does not include screening effects, so we think it is more reliable to fit the parameters to the dispersion curve of the band structure calculations. Taking $m^{*}=1.3 m$ for the smooth part of the effective mass, eqs. (A1,A3) give a constraint on the sum of $b_{1}$ and $b_{2}, b_{1}+b_{2}=18.7 \mathrm{eV}-\AA^{3}$.

The other result of the band theory we use is the energy gap $E(N)-E\left(N^{\prime}\right)$ at the $N$ point, which we take as $E(N)-E\left(N^{\prime}\right)=2.75 \mathrm{eV}$. Numbers close to this value were 
obtained in ref. $[6,10,15]$. The energy gap is related to the backward scattering $T$-matrix at the momentum $k_{N}$,

$$
E_{N} / 2=V_{110}=\left\langle-k_{N}|v| k_{N}\right\rangle=n\left(b_{0}+\left(b_{2}-b_{1}\right) k_{N}^{2}\right)
$$

We have considered a variety of parameter sets with these constraints, but we will show results for only three cases. Case I listed in Table I is a local interaction that fits the $E(N)-E\left(N^{\prime}\right)$ band gap, but has a unit effective mass. This pseudopotential will show the specific lattice effects. Case II adds a $p$-wave term so that both the smooth effective mass and the band gap are fit. Finally, case III gets an effective mass from a mixture of $s$ - and $p$-wave pseudopotentials to see what the effect is of this degree of freedom.

\section{RPA RESPONSE IN THE JELLIUM MODEL}

We start with the spherical jellium model of the electronic structure of alkali metal clusters. The model assumes a uniform density of positive background charge in a sphere of radius $R=(N)^{1 / 3} r_{0}$. The single-electron wave functions are obtained from the selfconsistent solution of the Kohn-Sham equation using the local density approximation for the exchange-correlation energy. The response is obtained from the linear response theory based on the same Hamiltonian, using the computer program of ref. [13].

The calculated single-electron density of states for the spherical cluster $\mathrm{Li}_{138}$ is shown in Fig. 2. The density parameter $r_{0}$ is taken at its bulk value, $r_{0}=3.25$. The bound states are of course discrete; to make the plot we have smeared out the delta functions in the density with the replacement $\delta\left(E-\epsilon_{i}\right) \rightarrow \pi \gamma /\left(\left(E-\epsilon_{i}\right)^{2}+(\gamma / 2)^{2}\right)$ Note the very strong shell effects among the bound states. The continuum has been discretized by imposing a boundary condition on the wave functions, $\psi(3 R / 2)=0$.

In Fig. 3 we show the dipole response of the cluster, expressed as a photon absorption cross section. The dashed line shows the independent particle response of the system, that is, without interactions between the electrons. This response is dominated by the low 
transitions just across the Fermi surface, which have large transition dipole moments. The solid curve shows the RPA response, treating the particle states in the continuum exactly rather than with the discretized spectrum of Fig. 2. The RPA strength is concentrated in a small region of excitation near $4 \mathrm{eV}$. This is the Mie resonance, shifted from its classical value, eq. (1), by about $10 \%$. As mentioned earlier, the shift can be accounted for mainly as a spill-out effect, that the effective radius of the electron sphere is slightly larger than $R$. We note that the RPA preserves the energy-weighted sum rules, and the predicted oscillator strength in the spectrum (to $15 \mathrm{eV}$ ) is within a few percent of the sum rule value of 138 .

We cannot use the same numerical technique to calculate the response of the system with ionic perturbations because that method requires spherical symmetry. Instead, we use a simplified treatment of the interaction as a separable function of the coordinates of the interacting particles. Specifically, we will take the electron-electron interaction to have the following dipole-dipole form,

$$
v_{L=1}\left(r_{1}, r_{2}\right)=\frac{e^{2} f\left(r_{1}\right) f\left(r_{2}\right) \hat{r}_{1} \cdot \hat{r}_{2}}{R^{3}}
$$

where $f(r)$ is the potential field of a surface dipole source,

$$
\begin{gathered}
f(r)=r \quad r<R \\
=\frac{R^{3}}{r^{2}} \quad r>R
\end{gathered}
$$

The dynamic polarizability $\alpha(\omega)$ is then given by an algebraic formula ${ }^{1}$,

$$
\alpha(\omega)=-\frac{\Pi_{0}(\omega)}{1-e^{2} \Pi_{0}(\omega) / R^{3}}
$$

where $\Pi_{0}$ is the independent electron polarization propagator,

$$
\mathrm{I}_{0}(\omega)=\sum_{p h} \frac{2\left(\epsilon_{p}-\epsilon_{h}\right)\langle p|f| h\rangle^{2}}{\omega^{2}-\left(\epsilon_{p}-\epsilon_{h}\right)^{2}}
$$

\footnotetext{
${ }^{1}$ This is not quite the dipole polarizability because the field $f$ differs somewhat from the dipole field.
} 
Here the sum is over occupied orbitals $h$ and empty orbitals $p$. In Fig. 4 we show the predicted response with the separable approximation, using the particle and hole wave functions and from the discrete basis spectrum of Fig. 2. We see that the position of the Mie peak is virtually the same as before, but its splitting is slightly different due to small shifts of the particle-hole energies. There is also some structure in the region of 5-7 eV, but this is spurious, due to the discretization of the single-electron states in the continuum. In the continuum RPA there is also strength above the Mie peak, but it is a smooth shoulder. In any case, the separable approximation appears to be adequate to discuss perturbations which would introduce shifts and broadenings of the observed magnitudes of about $1 \mathrm{eV}$.

\section{EFFECT OF THE IONIC POTENTIALS}

We now modify the single-electron wave functions and energies by adding the ionic potential to the single-electron Hamiltonian, and diagonalizing in the space of wave functions constructed from the pure jellium approximation. Computationally, we can only handle easily bases with dimensionalities in the range of 500. For a cluster of 100 atoms, this means that the total space is about ten times the occupied space, taking into account spin degeneracy. We truncate our basis at an excitation energy of $15 \mathrm{eV}$, which is about three times the Fermi energy. This would be a very restricted space for a conventional pseudopotential, but the contact pseudopotential is parameterized to be used in a small basis. We consider two structures for the positions of the ion cores: the usual bcc lattice characteristic of alkali metals at finite temperature, and a Mackey icosahedron which is sometimes favored for finite systems [16].

For the bcc lattice, we position the ions by centering the jellium sphere halfway between neighboring ions in the lattice. The ion potential eq. (2) is placed at all lattice points that fall within the sphere, i.e. that satisfy $r<R$. The number of such points happens to equal 138 exactly. 


\section{A. Single-electron density of states}

The single-electron density of states for the three pseudopotential models of Table I are shown in Fig. 5. All these potentials preserve the shell structure up to the Fermi energy, which may be seen from the strong oscillations of the level density for negative energies. If the $s$-wave potential were much stronger, the shell structure would disappear. So the empirical

evidence [14] for shell structure places some constraint on allowable contact potentials. It may also be seen from Fig. 5 that the width of the spectrum is larger for model I than the other two models. This is an immediate consequence of the effective mass, which has a value $1.3 \mathrm{~m}$ for models II and III, but is the bare mass for model I. The final observation to be made from the displayed level densities is the weakness of the band gap. There is no dip observable in the spectrum I and only a weak decrease in the level density in the band gap region for potentials II and III. We thus expect that the band effects will be unimportant for clusters as small as $\mathrm{N}=138$.

\section{B. Dipole response}

We now consider the effect of the ionic perturbation on the response of the cluster. We expect the resonance to be broadened by the ionic potential as well as shifted down in frequency. For the extended metal, it was noted empirically that the plasmon width scales roughly as the square of the band gap [17]. We can test this dependence as well as the absolute magnitude with our model. We note also that in a calculation of the $\sim 20 \mathrm{eV}$ resonance in the $\mathrm{C}_{60}$ molecule, the ionic cores considerably broadened the resonance from its width in a jellium model [18]. Fig. 6 shows the photon absorption strength function calculated with the perturbed single-particle wave functions, using the independent particle response and the RPA response. From the independent particle strength function, shown as the dashed line, it may be seen that the perturbation induces considerable strength in the region of the Mie resonance. From the RPA result, the solid curve, we see that the Mie 
resonance remains at the same position, but is much broadened. This is to be expected from the presence of the nearby strength in the independent particle response, implying increased coupling to the resonance. The fact that the Mie resonance is not shifted shows that the band-gap mechanism of effective mass enhancement is not an important effect in small clusters. This could have been anticipated by the results on the single-particle density of states, which did not show a band gap.

Next we consider the effect of the momentum-dependent ionic potentials. Fig. 7 shows the RPA response for the potentials II and III of Table I. We see a downward shift of the main peak. However, the strength is quite fragmented, so the total strength below $4 \mathrm{eV}$ is only $25 \%$ of the sum rule. We also show the data from ref. [2]. It seems that the strength is spread out too much, and the downward shift is not enough. It appears that the lattice is behaving more like a set of incoherent scatterers than a periodic array that induces a band structure. Our treatment of the bcc lattice, to include lattice sites only within the jellium sphere, undoubtedly gives a more irregular surface than the system would realize.

We conclude this section with a comment on the energy-weighted sum rule. It is satisfied in the jellium model, but the momentum-dependent ionic potentials modify the sum, decreasing it approximately by the factor $m / m^{*}$. Since the true Hamiltonian has no momentum dependence, the loss of the sum rule is an artifact of the pseudopotential treatment. There are other parts to the wave function, hidden in the pseudopotential treatment, that contribute to the response and would restore the sum rule. We can only expect that the pseudopotentials would describe the strength correctly in the low energy region. At energies much higher than the Mie resonance, there would be additional strength not contained in the theory with the momentum-dependent pseudopotential.

\section{Icosahedral lattice}

We consider now an icosahedral lattice which may behave differently because of a different band gap or because of its different surface. The nearest closest complete Mackey 
icosahedron to $N=138$ is the structure at $N=147$, which has 3 layers outside the central atom. For this case we start from the jellium model for $N=147$, keeping the density parameter the same as before, and add the ionic perturbations of the 147 sites in the icosahedron.

Fig. 8 shows the density of states of this structure with potential III. It is somewhat similar to the density of states of the bcc lattice, but the gap is more pronounced. Perhaps the smoother surface is more important than the details of the lattice arrangement. In Fig. 9 we show the response for the cluster $\mathrm{N}=147 \mathrm{using}$ the icosahedral lattice. The Mie resonance is somewhat sharper, but the main features of the bcc clusters are similar. The resonance is too broad and too high in energy as compared to experiment. Thus, the dipole response is only mildly sensitive to the details of the lattice. This is certainly not the case of much smaller clusters: in ref. [3] it is found that the structure of the dipole strength function can distinguish different structures for $N \leq 10$. And, of course, structural changes that affect the shape of the cluster will split the resonance along different principle axes.

\section{CONCLUSION}

We have found that the contact pseudopotentials which fit the band structure predict too much spreading of the Mie resonance in the finite cluster, and do not lower the mean frequency enough to fit the data. Evidently, the contact form of the pseudopotential is rather singular for extrapolating from band properties to small finite systems. Other treatments of the ionic potential have been recently proposed using a spherical average $[8,9]$ of the potential. This represents an opposite limit of completely ignoring scattering effects of the ionic potentials.

In ref. [8], Blundell and Guet use a realistic (norm-conserving) pseudopotential for ions and make an angular average to give a spherically symmetric potential. The single-electron potential is then rather smooth, having only radial variations from the non-uniform radial distribution of ions. The effective mass with such a potential treatment is very close to one, particularly with respect to transitions between states of high angular momentum, where 
the radial wave functions are practically unchanged. Nevertheless, this model succeeds in producing a large red-shift of the resonance, and the experimental energy of $\sim 3 \mathrm{eV}$ is reproduced. Since the effective mass is not responsible, our interpretation is that the prescription these authors used for the single-particle potential resulted in a larger well than in the usual jellium model, with the electron density extending farther out. A more diffuse surface and lower average density would lower the Mie resonance. Part of the observed red shift might be from this source, but better theory is necessary to ascertain the importance of this mechanism. We also note that since there is no explicit momentum dependence in their potential, the energy-weighted sum rule is preserved. This likely gives too much strength in the peak region, as compared to experiment.

Explicit use of the nonlocality of the pseudopotential is made in a model by Serra, et al. [9]. These authors decompose a norm-conserving pseudopotential into a local part and parts proportional to the square of the gradient operator and to the square of the angular momentum operator. The potential is averaged uniformly over the jellium sphere, and the response is calculated in LD-RPA. Good agreement is obtained for the position of the resonance. The effective mass in the interior is $m^{*}=1.54$, which contributes a $20 \%$ red-shift to the resonance. However, we do not understand how the effective mass comes out so high in this model. From the band structure calculations, the effective mass is in the range 1.3 when effects of the band edges are omitted. The strong perturbation at the band edge increases the effective mass to the higher value, but this perturbation would be absent in a spherically averaged approximation. In this model, the energy-weighted sum rule is decreased by the factor $m / m^{*}$, in better accord with the experimental data in the peak region.

In both models of ref. [9] and [8] the pseudopotential does not alter the width of the jellium resonance. This is to be expected, because the spherical averaging in these models smooths out the potential so that the wave functions are not strongly mixed, as they are in our model. Note that the width depends somewhat on the details of the jellium model. In [8] it is about the same as the data, $\Gamma \sim 1 \mathrm{eV}$, while in ref. [9] it is much narrower than the data. The difference may arise from the treatment of the exchange interaction; the resonance 
may be more collective when exchange is treated in the local density approximation than when it is calculated exactly.

\section{ACKNOWLEDGMENT}

We thank B. Mottelson for his contribution to Appendix A. We also thank A. Bulgac, C. Brechignac and D. Tomanek for discussions. This work was supported by the Department of Energy under Grant FG06-90ER-40561.

\section{APPENDIX A: CORE POLARIZABILITY EFFECT}

The effect of the ionic core polarizability on the Mie resonance can be estimated rather simply as follows ${ }^{2}$. We use the separable model, eq. (3-5) with $f$ the usual dipole operator. In addition to the contribution of the $N$ valence particles to $\Pi_{0}$, there will be a contribution from the polarization of the $Z$ electrons in each ionic core. We assume that all of the transition strength for associated with the core polarizability is concentrated at a particular energy $E_{c}$. Then we may make use of the energy weighted sum rule,

$$
\frac{Z N \hbar^{2}}{2 m}=\sum_{f}\langle i|z| f\rangle^{2}\left(E_{f}-E_{i}\right) \approx E_{c} D_{c}^{2}
$$

to write

$$
\Pi^{\text {core }} \equiv \sum_{f} \frac{2\langle i|z| f\rangle^{2}\left(E_{f}-E_{i}\right)}{\omega^{2}-\left(E_{f}-E_{i}\right)^{2}} \approx \frac{Z N \hbar^{2}}{m\left(\omega^{2}-E_{c}^{2}\right)} .
$$

In a similar spirit the valence particle polarizability is

$$
\Pi_{0}^{v a l}=\frac{N \hbar^{2}}{m\left(\omega^{2}-\epsilon_{p h}^{2}\right)}
$$

where $\epsilon_{p h}$ is an average particle-hole energy. The resonance frequencies are now found from the poles of the polarizability function. This yields

$$
\omega=\omega_{M}\left(1-\frac{Z \omega_{M}^{2}}{E_{c}^{2}}\right)^{1 / 2}
$$

\footnotetext{
${ }^{2}$ We thank B. Mottelson who contributed substantially to the material in this appendix.
} 
provided $\epsilon_{p h}$ can be neglected in comparison to $\omega_{M}$.

We present in Table II the core polarization effect calculated according to eq. (A.1). The Mie frequencies are obtained from eq. (1) and the bulk densities of the alkali metals. The core excitation energy is obtained from the polarizability sum $S_{-2}$ in Table II of ref. [19]. The effect is appreciable in the heavier metals, but is not as large as Pines obtained for the bulk plasmon [20]. For lithium metal we can certainly neglect the core polarizability.

\section{APPENDIX B: EFFECTIVE MASS ESTIMATE}

To estimate the effect of the average ion core potential on the effective mass, we consider a single lithium atom embedded in a jellium continuum and calculate resulting energy shift. The perturbation is the difference between the actual core potential and the jellium potential representing the single atom, i.e. a uniform charge density occupying a volume

corresponding to the atomic volume $4 \pi r_{0}^{3} / 3$. Taking this charge distribution to be spherical, the perturbation is

$$
\begin{gathered}
\Delta V=V_{L i}(r)+\frac{e^{2}}{r_{0}}\left(\frac{3}{2}-\frac{1}{2}\left(\frac{r}{r_{0}}\right)^{2}\right) \quad r<r_{0} \\
=V_{L i}(r)+\frac{e^{2}}{r} \quad r>r_{0}
\end{gathered}
$$

In practice, the core potential is Coulombic for distances larger than $r_{0}$, so the perturbation vanishes for larger distances.

The effect of a dilute medium of uncorrelated scatterers is to produce an effective potential $U$ for propagating particles in the medium,

$$
U(k)=n\langle k|T| k\rangle
$$

Here $k$ is the momentum of the particles, $n$ is the density of scatterers, and $\langle k|T| k\rangle$ is the forward scattering $T$-matrix. The free-particle $T$-matrix is defined in terms of the scattering phase shifts $\delta_{l}$ associated with the potential $\Delta V$ as

$$
\langle k|T| k\rangle=-\frac{2 \pi \hbar^{2}}{m k} \sum_{l}(2 l+1) e^{i \delta_{l}} \sin \delta_{l} .
$$


If the phase shift is small, which is necessary anyway for this treatment to be sensible, we can replace the factor $e^{i \delta_{l}} \sin \delta_{l}$ by the phase shift itself,

$$
\langle k|T| k\rangle \approx-\frac{2 \pi \hbar^{2}}{m k} \sum_{l}(2 l+1) \delta_{l}
$$

This gives a purely real energy shift ${ }^{3}$ in eq. (B.2). Numerically, the lithium ion core gives the phase shifts shown in the upper part of Fig. 10, taking potential the pseudopotential of ref. [11] for $V_{L i}$ and $r_{0}=6.14 \AA$. The lower part of Fig. 10 shows the corresponding $T$-matrices, calculated from eq. (3). The s-wave contribution is repulsive and roughly independent of momentum, but the $p$-wave part is attractive and increases rapidly with momentum. This produces an effective mass greater than one; from the formula

$$
\frac{1}{m^{*}}=\frac{1}{m}+\frac{1}{k} \frac{d U(k)}{d k}
$$

the effective mass is about $m^{*} \approx 1.3 m$ at the Fermi surface $\left(k \approx 1.1 A^{-1}\right)$.

${ }^{3}$ See also ref. [12]. 


\section{REFERENCES}

[1] For a review, see W.A. de Heer, Rev. Mod. Phys. 65 (1993) 611.

[2] C. Brechignac, Ph. Cahuzac, J. Leygnier, and A. Sarfati, Phys. Rev. Lett. 70 (1993) 2036.

[3] V. Bonacic-Koutecky, P. Fantucci, and J. Koutecky, Chem. Rev. 91 (1991) 1035.

[4] V. Bonacic-Koutecky, et al., Chem. Phys. Lett. 213 (1993) 522.

[5] M. Rasigni and G. Rasigni, J. Opt Soc. Am. 67 (1977) 54.

[6] W. Ching and J. Callaway, Phys. Rev. B9 (1974) 5115.

[7] A. Maiti and L.M. Falicov, Phys. Rev. A44 (1991) 4442.

[8] S.E. Blundell and C. Guet, Z. Phys. D28 (1993) 81.

[9] L. Serra, G.B. Bachelet, N. van Giai, and E. Lipparini, Phys. Rev. B48 (1993) 14708.

[10] A clear exposition is given by F.S. Ham, Phys. Rev. 128 (1962) 2524.

[11] N. Troulier and J.L. Martins, Phys. Rev. B43 (1991) 1993.

[12] N. Fukuda and R.G. Newton, Phys. Rev. 103 (1956) 1558.

[13] G. Bertsch, Comp. Phys. Com. 60 (1990) 247.

[14] C. Brechignac, Ph. Cahuzac, F. Carlier, et al., Phys. Rev. B47 (1993) 2271.

[15] D.A. Papaconstantopoulos, "Handbook of the Band Structure of Elemental Solids,", (Plenum, New York) 1986.

[16] O. Echt, K. Sattler, and E. Recknagel, Phys. Rev. Lett. 47 (1981) 1121.

[17] P.C. Gibbons and S.E. Schnatterly, Phys. Rev. B15 (1977) 2420.

[18] K. Yabana and G.F. Bertsch, Phy. Scrip. 48 (1993) 633. 
[19] U. Fano and J.W. Cooper, Rev. Mod. Phys. 40 (1968) 450.

[20] D. Pines, "Elementary Excitations in Solids", Benjamin, 1964, p. 209. 


\section{TABLES}

TABLE I. Parameters of the ion effective potential, eq. (2)

\begin{tabular}{lr|rrr}
\hline \hline & & $\begin{array}{r}b_{0} \\
\left(\mathrm{eV}-\AA^{3}\right)\end{array}$ & $\begin{array}{r}b_{1} \\
\left(\mathrm{eV}-\AA^{5}\right)\end{array}$ & $\begin{array}{c}b_{2} \\
\left(\mathrm{eV}-\AA^{5}\right)\end{array}$ \\
& $T$-matrix & 8 & 20 & -2 \\
I & local & 29.8 & 0 & 0 \\
II & $p$-wave & -0.5 & 18.7 & 0 \\
III & $s$-p-wave & 29.8 & 9.4 & 9.4 \\
\hline \hline
\end{tabular}

TABLE II. Core polarizability correction to Mie resonance frequency, eq. (A.1).

\begin{tabular}{l|rrrr}
\hline Element & $\omega_{M}$ & $Z$ & $E_{c}$ & $\Delta \omega$ \\
& $(\mathrm{eV})$ & & $(\mathrm{eV})$ & \\
\hline $\mathrm{Li}$ & 2 & 4.70 & 32.7 & $-2 \%$ \\
$\mathrm{Na}$ & 3.45 & 10 & 52.7 & $-2 \%$ \\
$\mathrm{~K}$ & 2.54 & 18 & 34.7 & $-5 \%$ \\
$\mathrm{Rb}$ & 2.31 & 36 & 39.9 & $-6 \%$ \\
$\mathrm{Cs}$ & 2.08 & 54 & 38.3 & $-8 \%$ \\
\hline \hline
\end{tabular}




\section{FIGURES}

FIG. 1. Ion core effects on electron energies showing a band gap at the edge of a Brillouin zone. The parameters are appropriate to (bcc) lithium metal in the $\Gamma N$ direction.

FIG. 2. Single particle density of states in the LDA jellium model of the cluster $\mathrm{Li}_{138}$. The Fermi energy is indicated by the arrow.

FIG. 3. Dipole response of the cluster $\mathrm{Li}_{138}$. Solid line shows the response in RPA, and the dashed line shows the independent particle response.

FIG. 4. Dipole response of $\mathrm{Li}_{138}$, using the separable approximation, eq. (3), and the discrete basis of Fig. 2.

FIG. 5. Single-particle density of states with the ionic potentials I, II and III from Table 1.

FIG. 6. Dipole response with potential I, the local potential. The dashed curve is the independent particle response and the solid is the RPA. The data is from ref. [2].

FIG. 7. Dipole response with momentum-dependent ionic potentials. The data is from ref. [2]. Curves are labelled by the ionic potential used.

FIG. 8. Single-electron density of states for $\mathrm{Li}_{147}$ with icosahedral ionic perturbation.

FIG. 9. Dipole response of $\mathrm{Li}_{147}$ (ico), using ion potential III. The data is from ref. [2].

FIG. 10. Ionic core scattering in jellium. The upper part shows the $s$ - and $p$-wave scattering phase shifts associated with a lithium ionic potential defined in eq. (2). The lower part shows the corresponding $T$-matrix from eq. (B.3). 
Figl

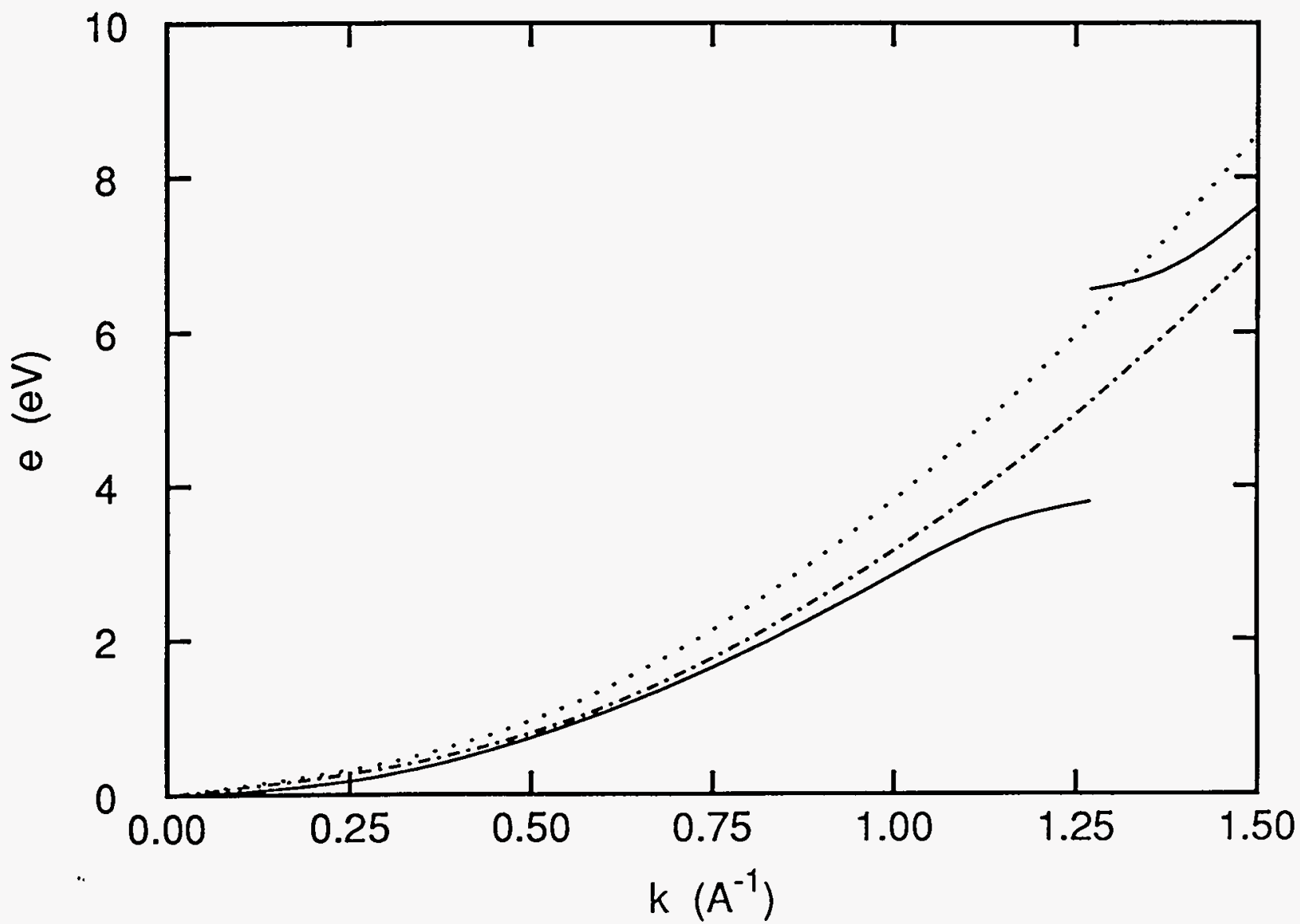




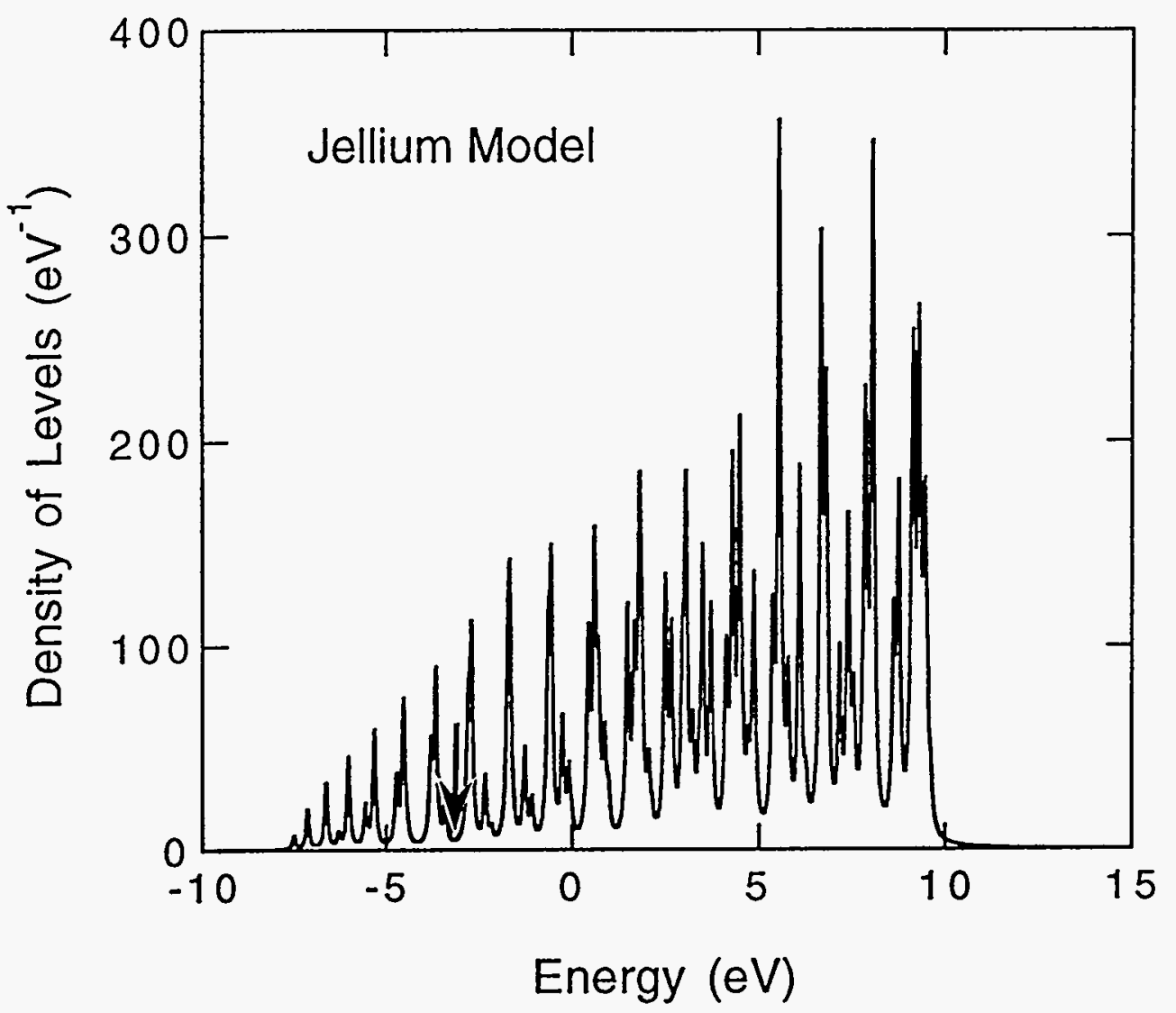

Fig 2 


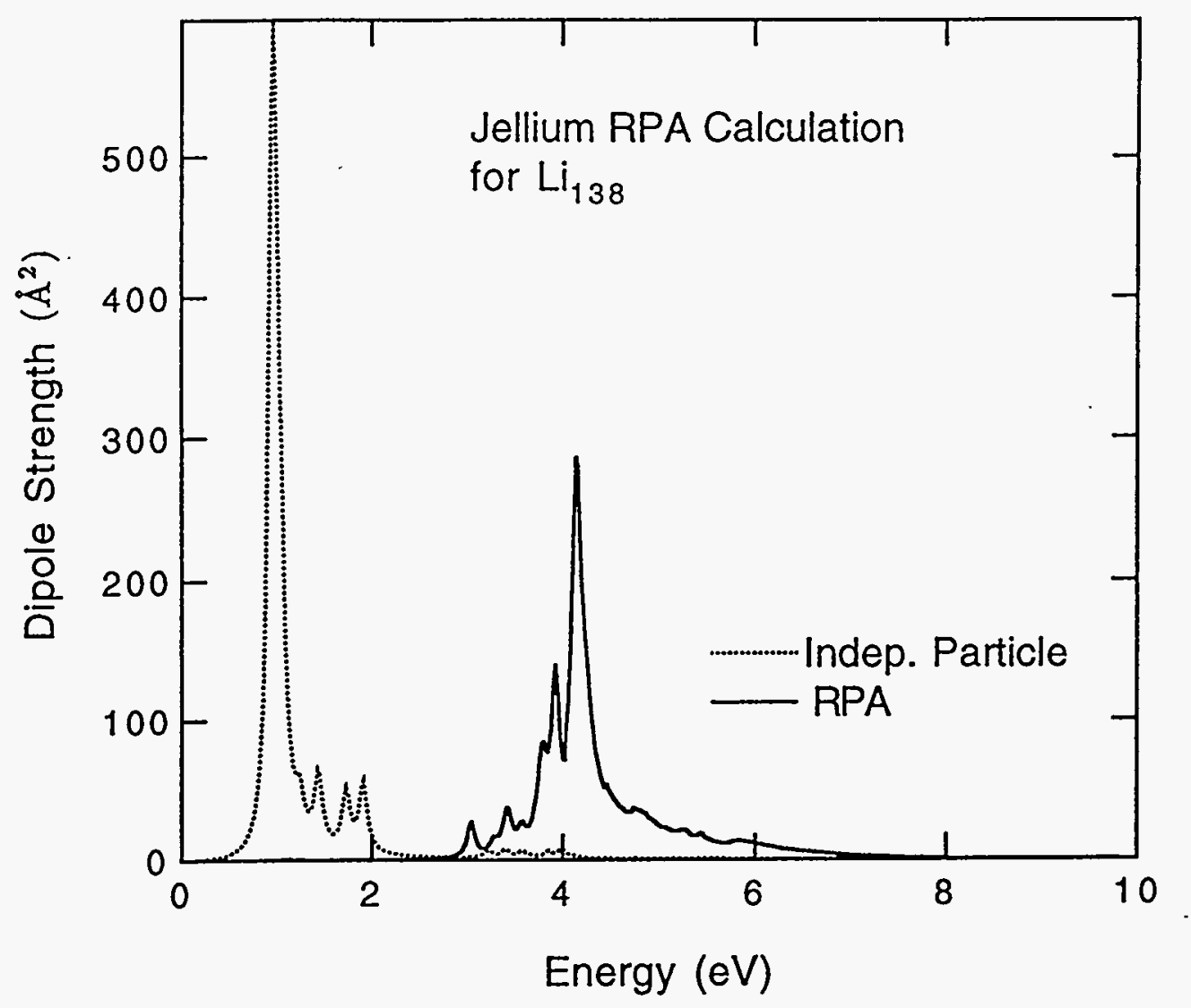

Fin. 3 


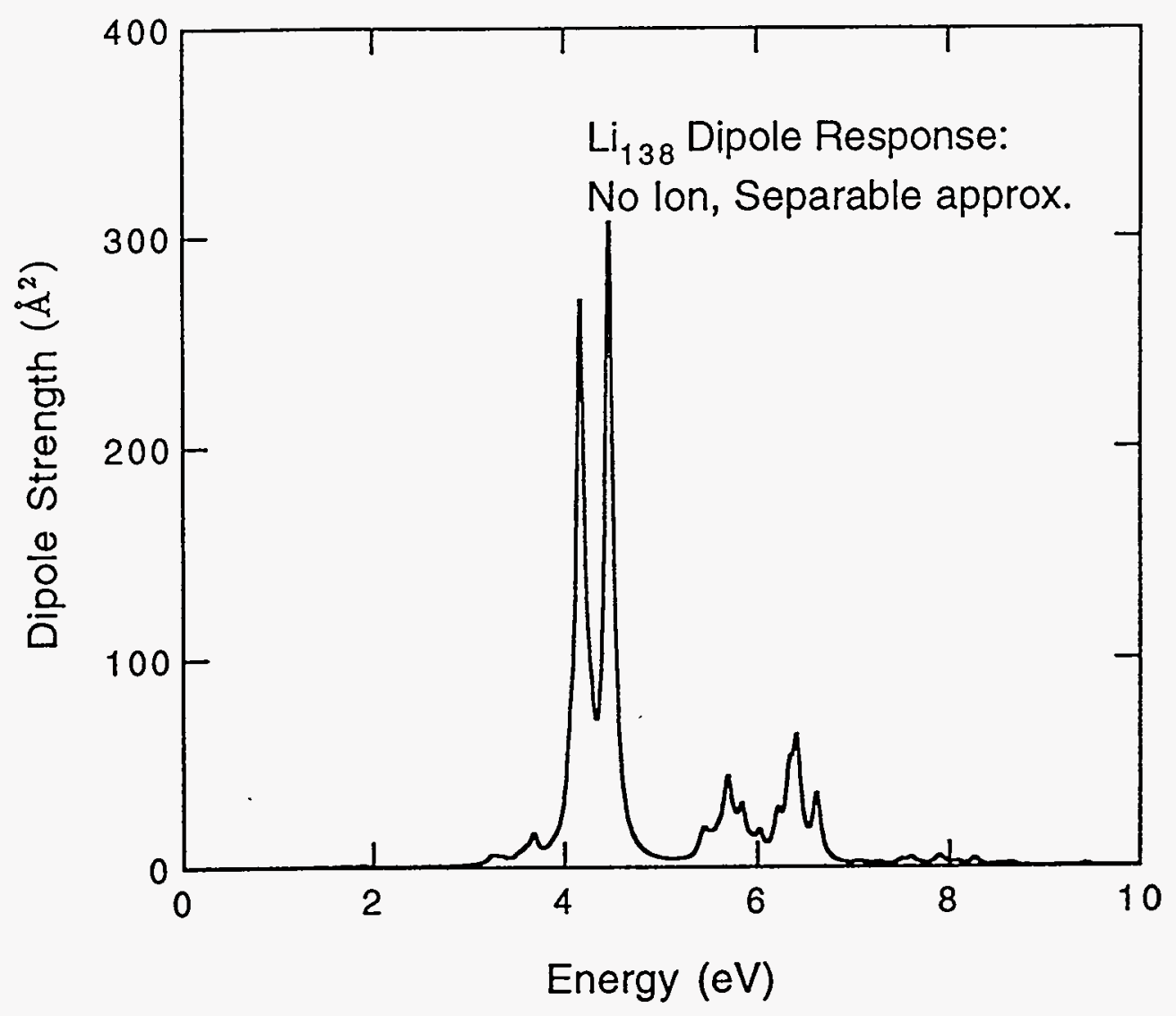

Fig. 4 


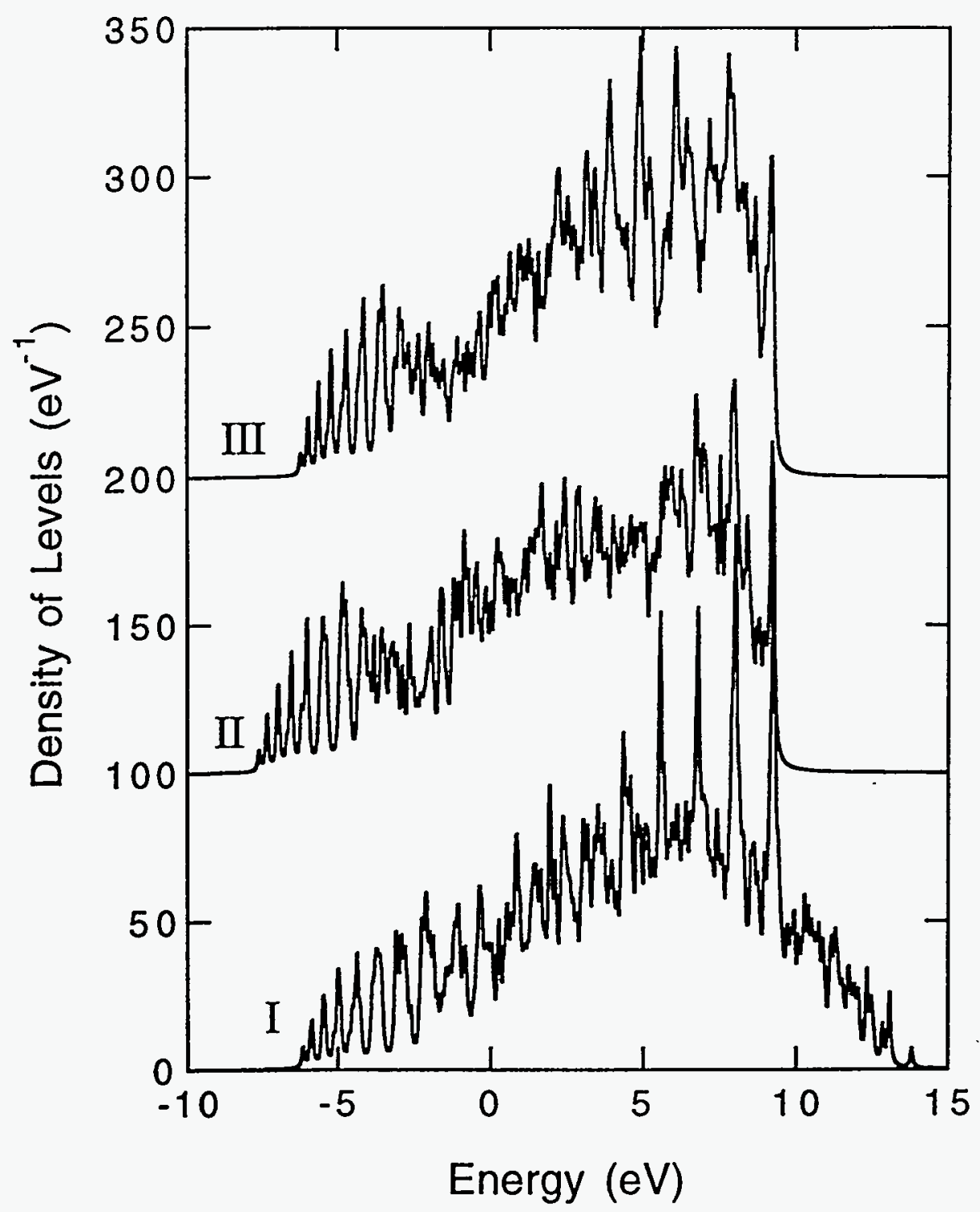

Fig 5 


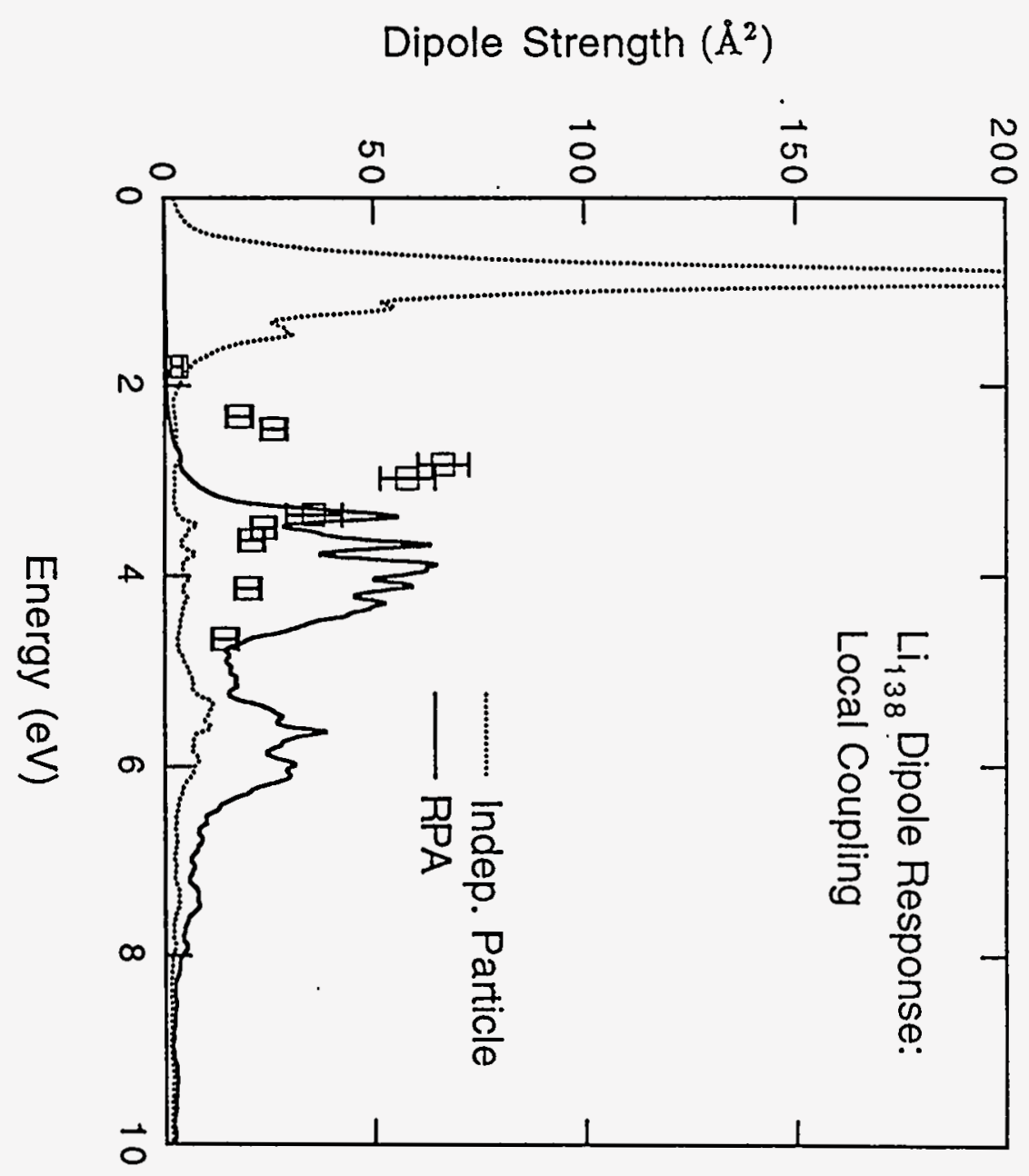




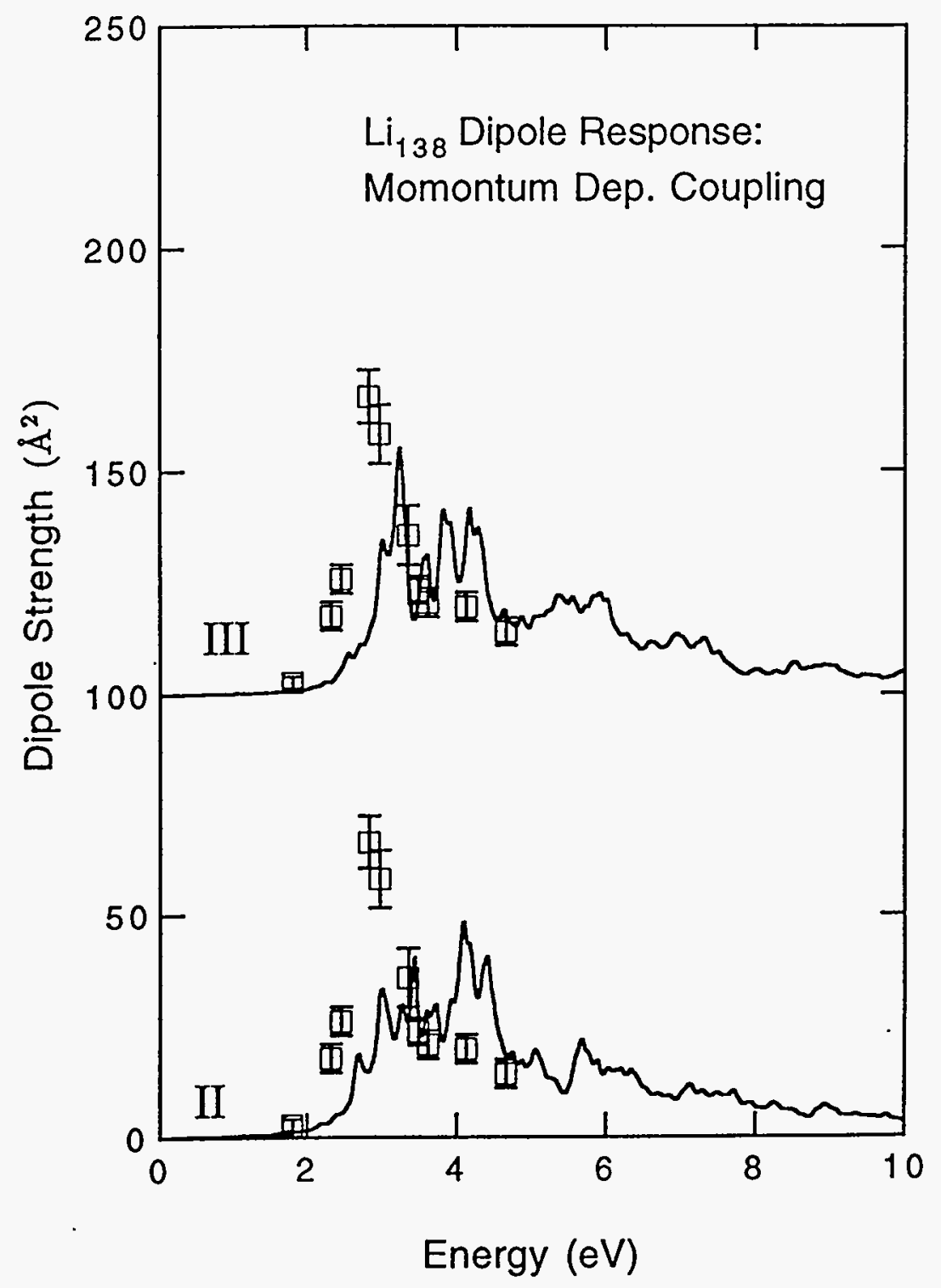

Fig. 7 


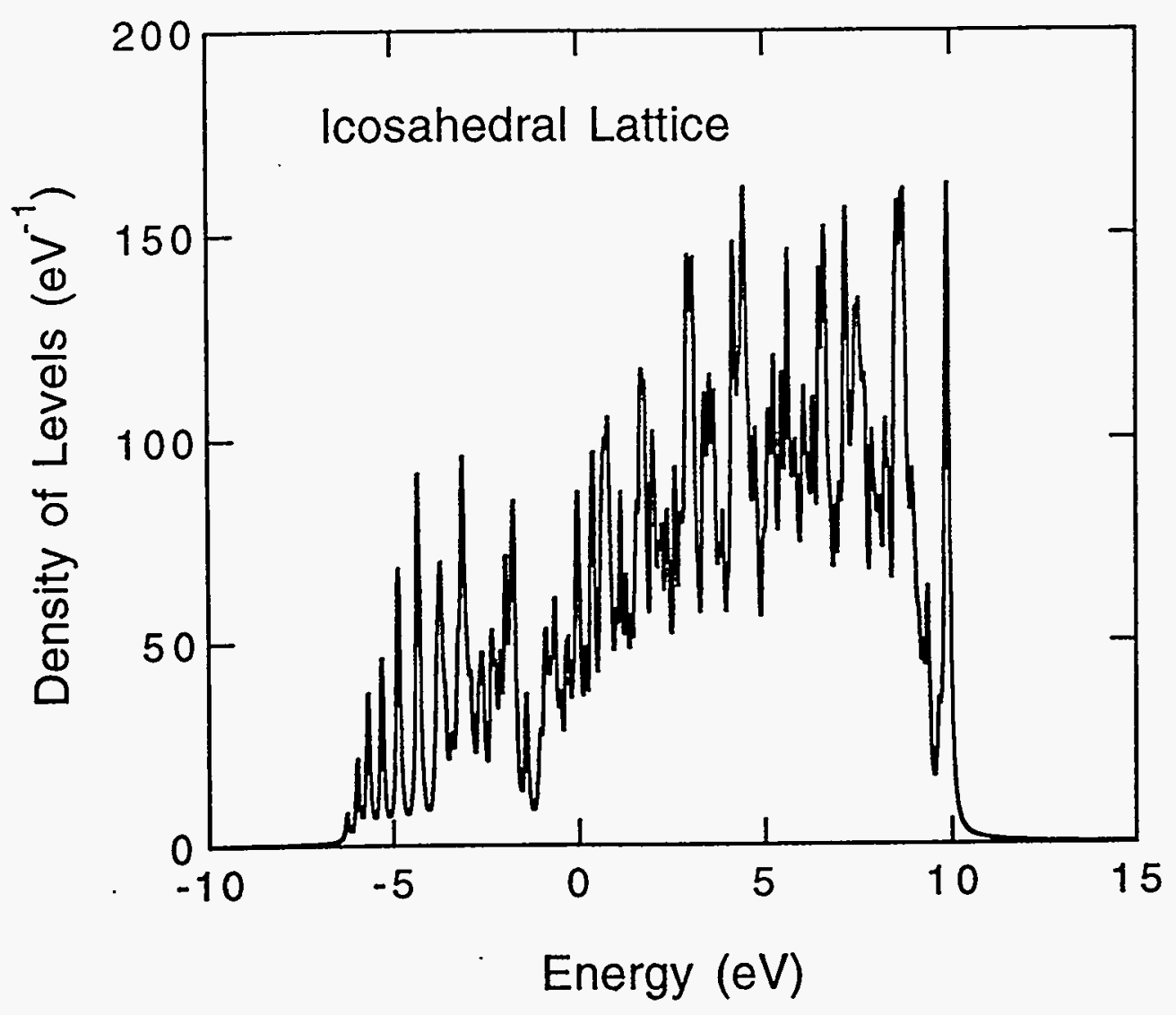

Fig. ${ }^{8}$ 


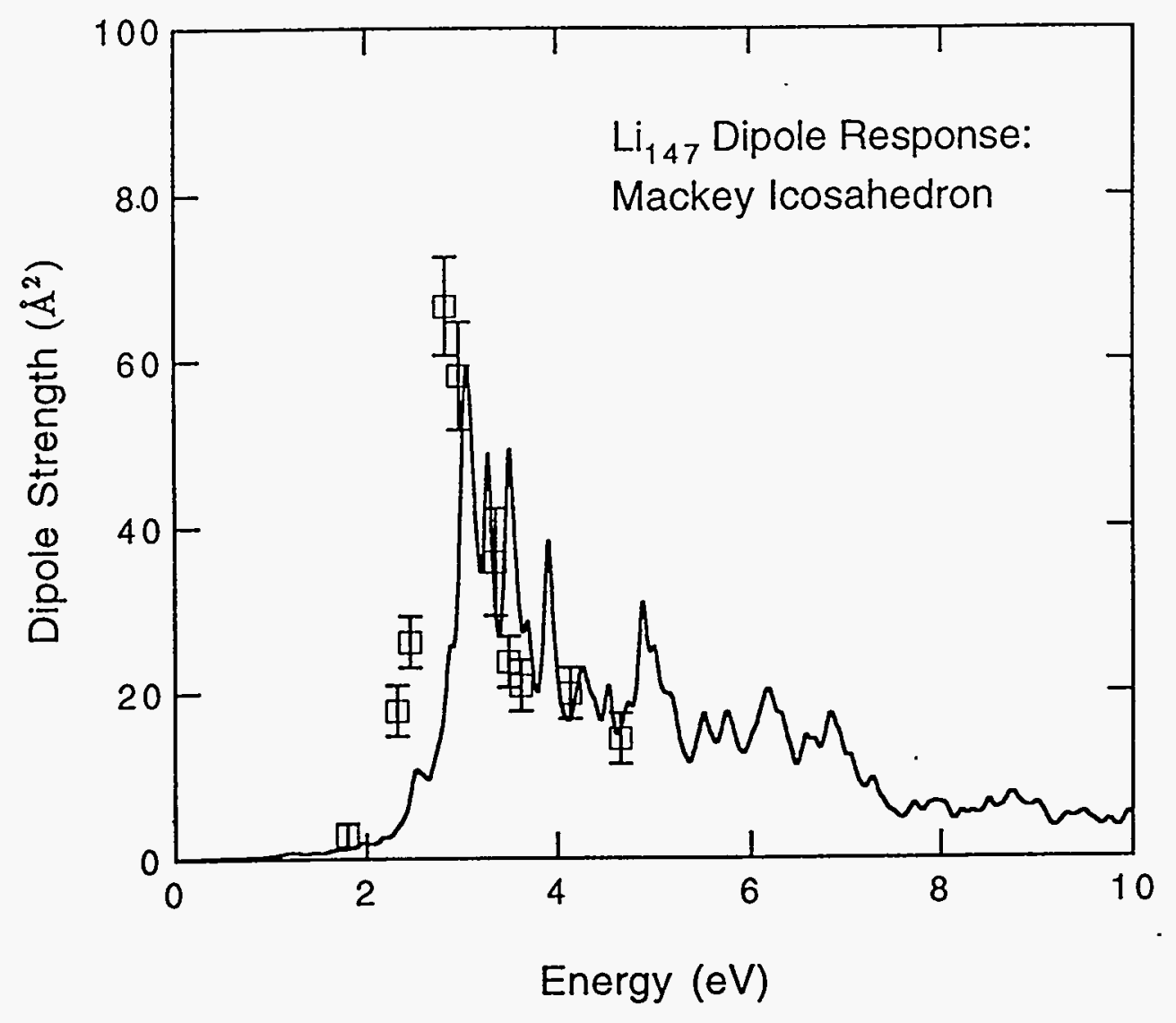

Fig. 9 
fig. 10.

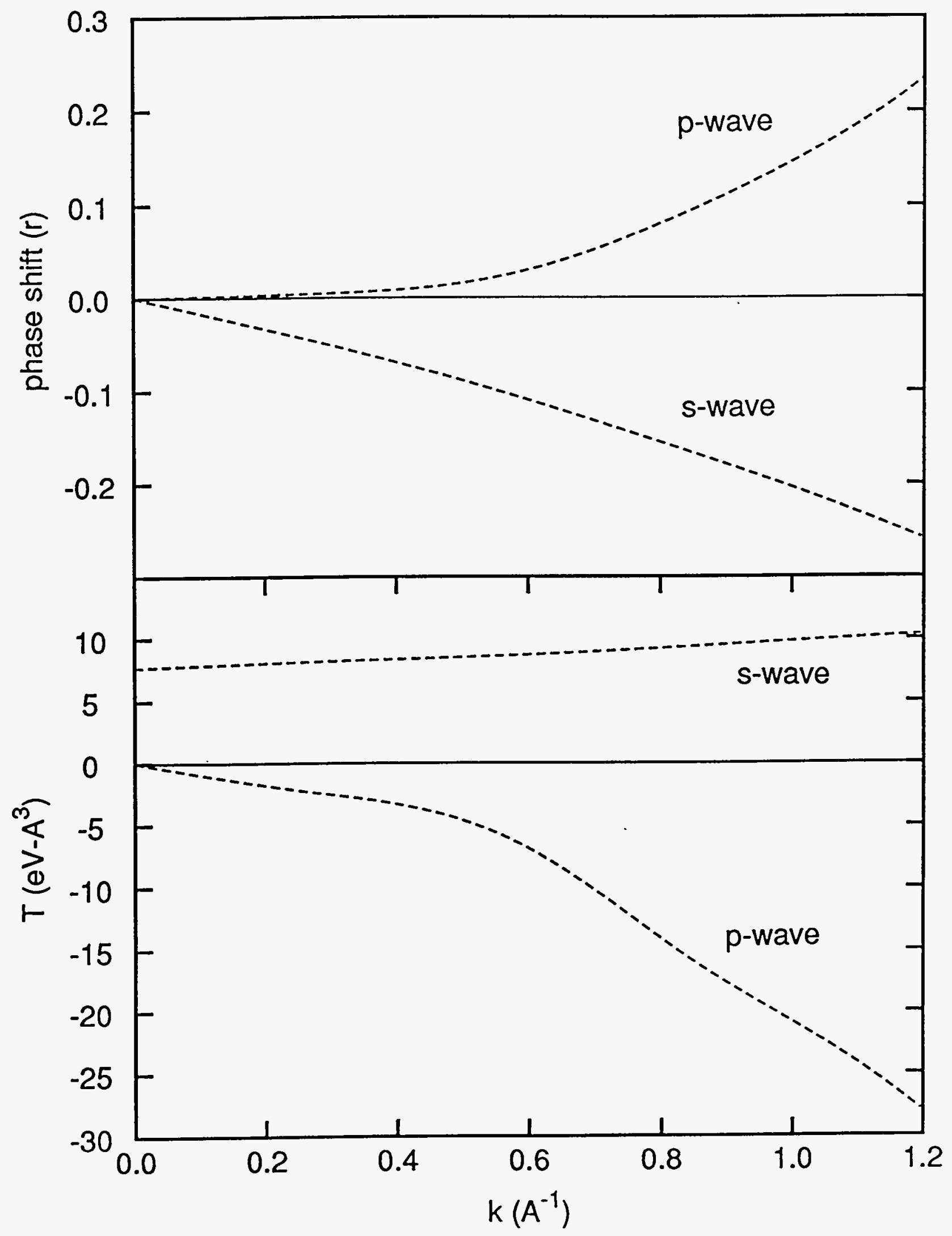

